\title{
Mobilizing Primary Health Care: Cuba's Powerful Weapon against COVID-19
}

\author{
Tania L. Aguilar-Guerra MD MS and Gail Reed MS
}

A strong foundation of primary care is critical to the health system and is particularly important during pandemics like COVID-19. Primary care practices should be a natural fit for triaging, testing, treating, and educating patients.

-Corinne Lewis, Shanoor Seervai, Tanya Shah, Melinda K. Abrams, and Laurie Zephyrin MD The Commonwealth Fund, April 22, 2020[1]

This thoughtful observation by The Commonwealth Fund writers seems to describe what we were finding in Cuban neighborhood after neighborhood, as we interviewed for this story: the backbone of Cuba's universal public health system is also the backbone of its response to the coronavirus pandemic. Primary health care. And the results are beginning to emerge: as of April 30, 2020, through primary-care case detection and contact tracing, sources for $85.7 \%$ of the 1537 COVID-19 cases to date had been identified, essential for flattening the curve.[2] On May 1, the New York Times reported Cuba at 13 cases per 100,000 population and less than 1 death per 100,000; well below rates in the USA, Italy, Spain, France, the UK, Canada and Brazil, among others.[3]

In the Cuban case, primary health care (PHC) takes the form of 449 multispecialty community polyclinics, each serving as a hub for 15 to 40 neighborhood family doctor-and-nurse offices. Catchment populations are defined by geographic area, meaning that each doctor-nurse team is responsible for prevention, health promotion, patient care and rehabilitation of up to 1200 people (300 families); and the polyclinic's total population usually varies from 20,000 to over 40,000, depending on density. Cuba is 75\% urban, but in rural areas, longer distances mean responsibility for fewer patients.

Since the late 1980s, $100 \%$ of Cubans have had free access to family doctors and nurses, with more than 10,000 offices dotting the island today. These health professionals are embedded in the neighborhood, the doctor living above a modest office, exam room and waiting area. Family nurses often come from the neighborhoods they serve.[4]

Nearly all polyclinics have earned national accreditation to teach medical, nursing and allied health students, and provide a setting for curricula that include large doses of direct patient engagement and community-based learning. Polyclinic professionals are also responsible for local maternity homes, pharmacies, seniors' clubs and other community institutions... and in the case of COVID-19, for just about any local business, church, hotel, school, factory or even national ministry in their health area.

\section{COVID-19: Building on the Strengths of Primary Health Care}

The first three cases of COVID-19 diagnosed in Cuba on March 11,2020 , caught no one in the health system by surprise. In fact, since January, health authorities and government had mapped out an intersectoral approach to prepare for the potential onslaught.[5] Working at the time with few real-time polymerase chain reaction (RT-PCR) tests-made available by PAHO to the Pedro Kourí Tropical Medicine Institute, IPK_-and without early access to rapid tests, massive testing was clearly not in the cards as a first strategic option. However, primary health care was: the universal subsystem already at work in neighborhoods across the country was destined to become the health system's front line barrier and first line of attack against the novel coronavirus.

What has made it so? Insights we gleaned from dozens of interviews and reports are summed up as follows:

1) Cuba's is a single, universal, public health system with the ability to mobilize and adapt its considerable human and logistical resources to confront new situations, despite material resource constraints. The largest labor force in the system is working in primary health care, where it is highly structured and experienced for both training and care.

2) The primary health care subsystem was already operating under protocols for continuous community health assessments (public health-epidemiology), as well as individual and family health evaluations (dispensarización, or Continuous Assessment and Risk Evaluation, CARE). In addition, even medical students have experience in door-to-door case finding for infectious patients, given their participation in quelling dengue outbreaks in Cuba over the years.

3) Communication is well organized both horizontally within primary health care and vertically with other levels of the health system, permitting data sharing and quick implementation of new measures and activities as necessary.

4) Family doctors and nurses are committed teams, used to being available 24 hours a day (although their time is usually respected), and to making regular house calls as well as receiving patients for office visits.

5) Family doctors and nurses are literally next door, and have gained a reputation over $\mathbf{4 0}$ years as trustworthy healthcare providers: when they talk, people listen and are apt to follow their lead. People feel safer knowing they are around the corner or up the hill. These health care providers know their communities, and in turn count on their communities' cooperation and support.

\section{How these factors play out in a primary care strategy for COVID-19}

Single, universal, adaptable health system, capable of COVID-19 training and mobilization of primary-care personnel:

Dr Mayra García is director of the 19 de Abril University Polyclinic in the heart of Havana, which is responsible for 25 family doctorand-nurse offices and for 25,304 residents in its health area. It 


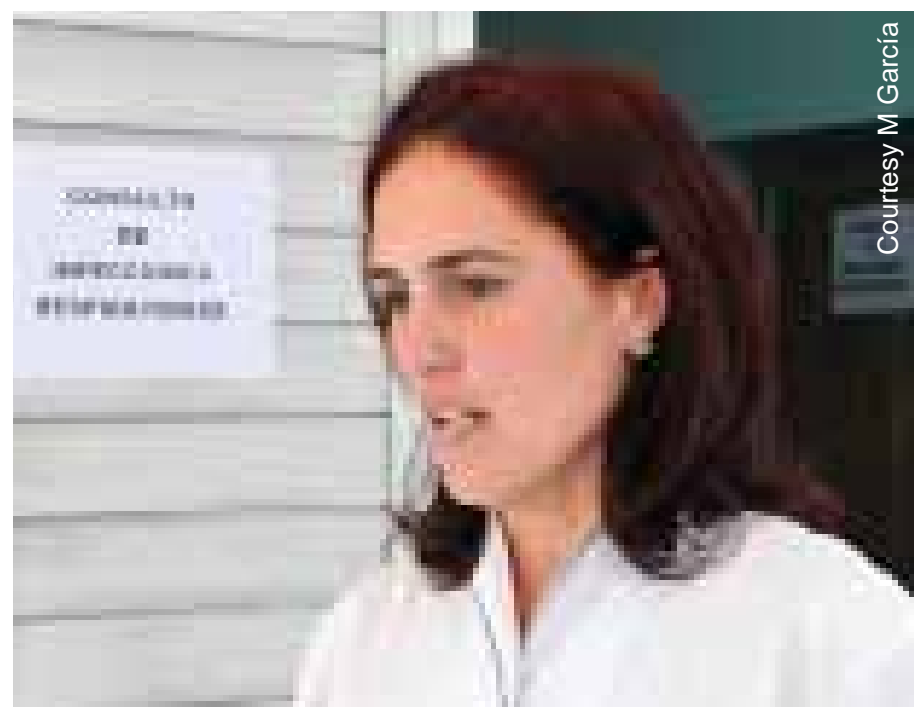

Dr Mayra García: Training included "anyone who might come in contact with a patient, including all support staff."

also has one of the country's oldest populations, with more than $30 \%$ over 60 years of age. She says special COVID-19 training of the health workforce was key to mobilization well before March's first cases were identified.

"Training was three tiered," she explains. "Everyone in the health system was trained on this coronavirus, how it was transmitted, and what was happening globally with its spread. First, we had a course at IPK involving professionals selected from each province, and then they returned home to share this new knowledge locally, the second tier. Here in Havana, this resulted in the provincial health department creating a group to train personnel. They trained directors of hospitals, polyclinics and the like, area by area, because Havana has 82 polyclinics, each with a fair number of family doctor-and-nurse offices. Then they went on to the third tier: training for family doctors and nurses themselves, lab and radiology technicians, administrative personnel, and also housekeeping staff, ambulance drivers and orderlies. Anyone who might come in contact with a patient, including all support staff. This last is key, I think, because people are always thinking about doctors and nurses, but what about other health personnel? They need to be just as aware and just as protected."

But Dr García says the training didn't stop with the health sector: the polyclinic was responsible for more. "Each polyclinic's staff went out to train people in the workplaces in their geographic health area, including small business owners, such as people renting their homes or managing private child care facilities. Family doctors, for example, mapped the houses that were being rented (especially those to foreigners) and wherever children were being cared for, to explain COVID-19 health measures and protocols so they could better protect themselves and those in their homes and businesses, learning what to do, how to recognize symptoms, and so on."

Dr Rubén García, who has spent the last 22 years as a family physician in various primary-care responsibilities, is now vice director for medical care in Havana's Plaza Municipality, where the 19 de Abril polyclinic is located. He notes that reorganization of primary care activities started early, beginning at the top: "All of the supervisors-whether nurses, doctors or program directors, who had been trained as family doctors and nurses-went to family doctor-and-nurse offices to reinforce them. We left very few at the top of the primary care subsystem in the municipality. We also involved dentists and physical therapists, whose workload was beginning to diminish as a result of stay-at-home guidelines. And in the case of polyclinics like 19 de Abril, we transferred some personnel from other areas to theirs, to even out our capabilities across the network."

He notes that more family medicine specialists, dentists and nurses were posted in local hotels, to provide 24-hour case detection and medical care to foreigners still residing there. At one point, some 320 guests were lodged in one hotel, the responsibility of a single polyclinic.

The polyclinics also reorganized services, in order to isolate from the rest of patient care the areas for people with respiratory symptoms, mainly those referred by family physicians but also some walk-ins to emergency services. Explains Dr Mayra García: "One of the first orders we received was to create different areas for evaluating people with symptoms of acute respiratory infections (ARIs), including both doctor office areas and urgent care. So we restructured to keep those patients separate from others. The new areas are staffed $24 / 7$ to assess all ARI patients who come in, as is the polyclinic 'command center,' where doctors finally determine the course of care or remittance of patients with respiratory or other symptoms of COVID-19."

Regularly scheduled appointments at polyclinics or with family doctors were postponed whenever possible, and prioritized patients such as older adults with chronic conditions or pregnant women were most often visited at home, rather than seen in offices. This reorganization of family doctor-and-nurse time then enabled these duos to head up newly organized efforts, including daily active case detection and contact tracing in their neighborhoods.

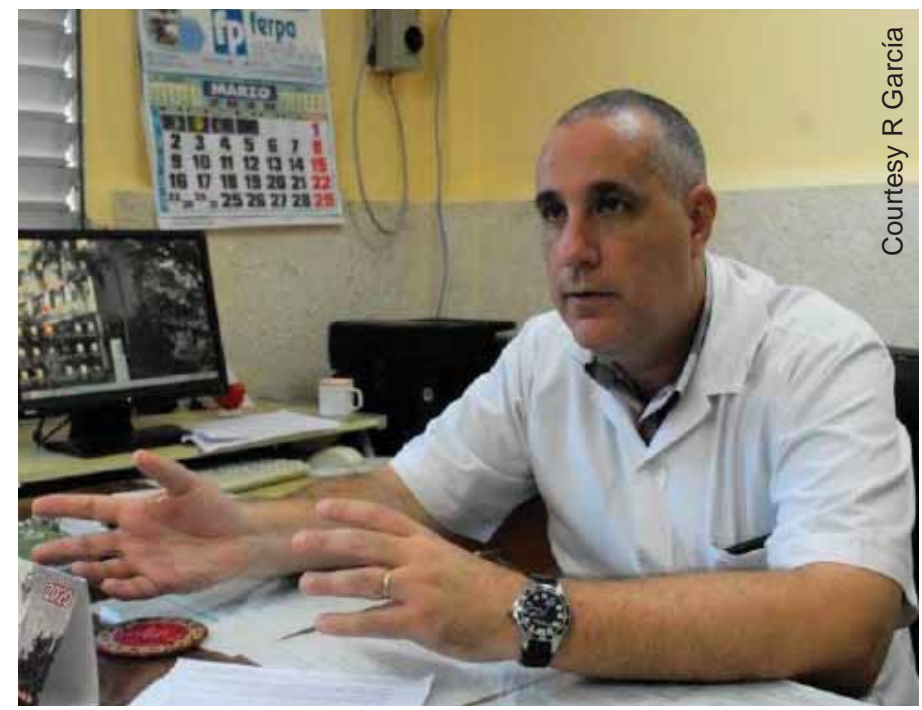

Dr Rubén García: "All of the supervisors...trained as family doctors and nurses... went to family doctor-and-nurse offices to reinforce them." 
Primary care already operating under protocols for continuous community, family and individual health assessments:

Two essential tools of Cuban primary care are the community health assessment and the individualized CARE model. The first makes use of a medical training that melds clinical medicine with public health, by assessing the general health-including environmental issues and social conditions-of a neighborhood annually by family doctors and then by the polyclinic. The CARE model goes more deeply into family and individual medical histories, categorizing each person into one of four groups: apparently healthy, with risk factors for disease, ill, and finally in recovery or rehabilitation. Risks include such key factors as smoking and overweight or obesity. III includes acute as well as chronic conditions, such as hypertension and diabetes. This information is collected by family doctors and nurses from the people they serve in their neighborhood, for whom they keep paper medical records that also include items such as well-baby visits, vaccinations, pregnancies and so forth.

Dr Marta Gálvez, family physician with a master's degree in geriatrics, has been in her neighborhood family doctor-and-nurse office \#9 of the Plaza University Polyclinic for the past 29 years... except for those times when she volunteered abroad in places as far away as Namibia. She says the CARE model is fundamental: "The first thing any self-respecting doctor must know is the health situation of the population she serves. You need to know what to look out for, what you need to prevent, what you need to promote. The main goal of a primary care physician is health promotion and prevention of diseases, so you have to know your community to design a strategy that suits their needs. CARE is a vital tool: it's why I know that I have 658 older adults in a total population of 1093 people, and 42 of the elderly live alone. That's one of my biggest challenges, the aging of the people in my neighborhood."

Dr Alejandro Fadragas, family physician with master's degrees in infectious diseases and sexology, has served in office \#8 in his Plaza University Polyclinic neighborhood for 18 years. "The CARE model also automatically alerts us to people who are more susceptible to respiratory infections, the people whose chronic diseases are the risk factors most commonly associated with complications in COVID-19 patients. This way, we already know who they are," he says.

With the experience of applying such protocols, primary health care teams were prepared to incorporate new actions into their daily routines. Since they already made house calls most afternoons, it was not a stretch to turn these visits into the single most important tool since the early days of surveillance: active case detection.[6] The protocols in place for COVID-19 call for daily active case detection in every Cuban neighborhood, with some 28,000 medical students joining the effort led by family doctors and nurses.

Dr Gálvez explains how this works: "Mainly we're actively looking for respiratory symptoms, active case detection, and if these appear, then, depending on how the symptoms are classified, we follow one or another protocol. For example, when we find someone with symptoms, we take an epidemiological survey, based on a very detailed questionnaire; we also make a thorough physical exam and a comprehensive evaluation. We use all this to decide what conduct to follow.

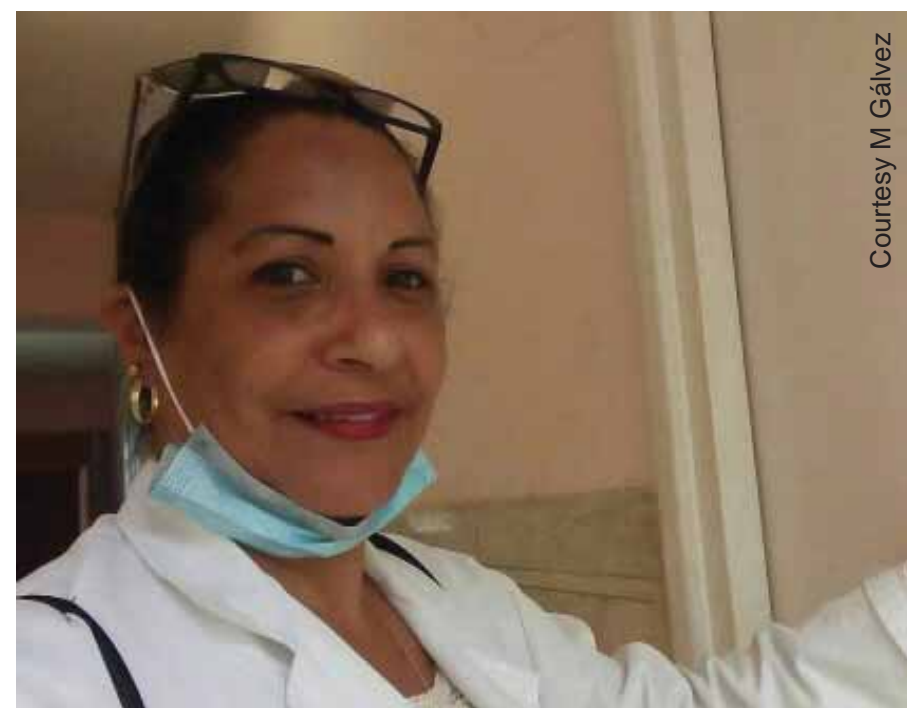

Dr Marta Gálvez: "The first thing any self-respecting doctor must know is the health situation of the population she serves."

It also enables us to survey $100 \%$ of our population on a daily basis, starting with the medical students every morning, household by household."

If a person is suspected as having COVID-19, they are remitted to their polyclinic for evaluation, and if physicians there determine that indeed they have a suspected case, then the patient is first isolated in the polyclinic itself and then sent to one of the isolation centers now set up in every municipality in the country, for a period of at least 14 days (where they receive rapid tests). If the case appears to be another respiratory illness, the person returns home, but must stay there for at least 14 days, followed in primary care.

Dr Gálvez emphasizes: "The importance of this whole process of active detection at the primary care level is so that hospitals are reserved for those patients who really need them: thus, those patients with COVID-19 have the ICU bed they need, the attention for any complication that might present. But the rest of the acute respiratory patients can be cared for at our level. That way, even as patient numbers may increase, we have a better chance of giving everyone the care they need."

But active case detection, evaluation, temporary isolation and referrals aren't the only responsibilities of primary care professionals during the COVID-19 pandemic: they are also entrusted with the equally important job of contact tracing for all suspected or confirmed cases. Personnel at hospitals specially selected for COVID-19 patients carry out an epidemiological survey of their own, to identify contacts the patient may have had.

Dr Gálvez says this part can be stressful: "Imagine! You have to decide among competing priorities. Just now, for example, I got a call from the polyclinic director, who says there are three people in my neighborhood who had contact with a confirmed case. So I need to visit them immediately. Then there is another patient who is not feeling well. The nurse and I have to juggle things on an hourly basis."

Josefa Nietos has spent 34 of her 45 years as a nurse in primary care, and she was a founder of the family doctor-and-nurse pro- 
gram in the Plaza Polyclinic health area, where she works in family doctor-and-nurse office \#11. "Yesterday, we saw the first family in our neighborhood that had contact with a confirmed COVID-19 case. The family was asymptomatic, and we remitted them to the polyclinic so they could be sent on to an isolation center for the required 14 days. And then their home was disinfected."

Home disinfection of contacts and suspected cases is a lesser-known aspect of primary care tasks, and is carried out by a "rapid response" team, usually made up of the polyclinic director and two vice directors, who disinfect households with family members. This is carried out with various disinfecting solutions, for surfaces and even for staircase railings in apartment buildings. If need be, family doctors and nurses pitch in.

Protection of health workers, and the apparently healthy people they come in contact with, is another concern in primary health care. Dr García notes that the workers at a hotel in her polyclinic area are checked daily by nursing and medical staff assigned there, "because the foreigners have been lodged there for over 21 days without becoming ill, so if they're infected now, it's our responsibility." Disinfectants, gloves, masks and other protective gear are provided by the polyclinics; Dr Gálvez says they change masks every four hours, wash them, boil and then iron them for re-use; and they disinfect family doctor-and-nurse offices and their homes daily.

Dr Fadragas notes that once at home, he leaves his shoes outside, removes all his clothes, separates them for washing, and jumps in the shower. "I don't deny that I'm worried about me or my family becoming infected," he says. "So I wish we had even more protection."

\section{A vital communications network involving primary care:}

As was alluded to by Dr Gálvez, polyclinic "command centers" are in touch throughout the municipality, province and even country, to alert for contact tracing and possible cases. In the early phase, when travelers were still flying into Cuban airports, this also involved rapid communication from airport and port medical personnel, who received the health questionnaires filled out by each passenger. These included the address where each was headed in the country, and local family doctors were charged with follow up for all.

Essential communications lines with hospitals have also been set up: when COVID-19 patients are discharged, they require follow-up in primary care for the next 14 days, and then must be cleared by a RT-PCR in order to receive an "epidemiological discharge." All this falls on local polyclinics, family doctors and nurses.

\section{Primary health care providers committed, used to 24/7 res- ponsibilities:}

There is no doubt that the heavy lifting required in primary care during COVID-19 is testing not only professional prowess, experience and organization, but also commitment. These health workers must be on the ball, ready to move and to make decisions that can change the course of the spread of the disease.

Dr Gálvez notes: “I have lost track of time; I don't know when I'm not at work. Right now, you have to dedicate yourself entirely to the job. Because depending on what you do now, later you'll have less work to do, because fewer people will be getting sick. It's prevention and health promotion that ensures our communities' health, after all."

"There are days we finish at 10 o'clock at night, but we can't rest now. You feel a tremendous sense of responsibility," says Dr Fadragas, "We're right here, every day, in touch with the population, where they live, where we need to make sure things are done right to protect everyone. We know we're on the front lines, and we're the most effective ones there. We're the ones who have the main job of educating people, especially during our 'rounds' of active case finding, to increase awareness of risk perception. It's not an easy task, but we know we have to do it and do it well."

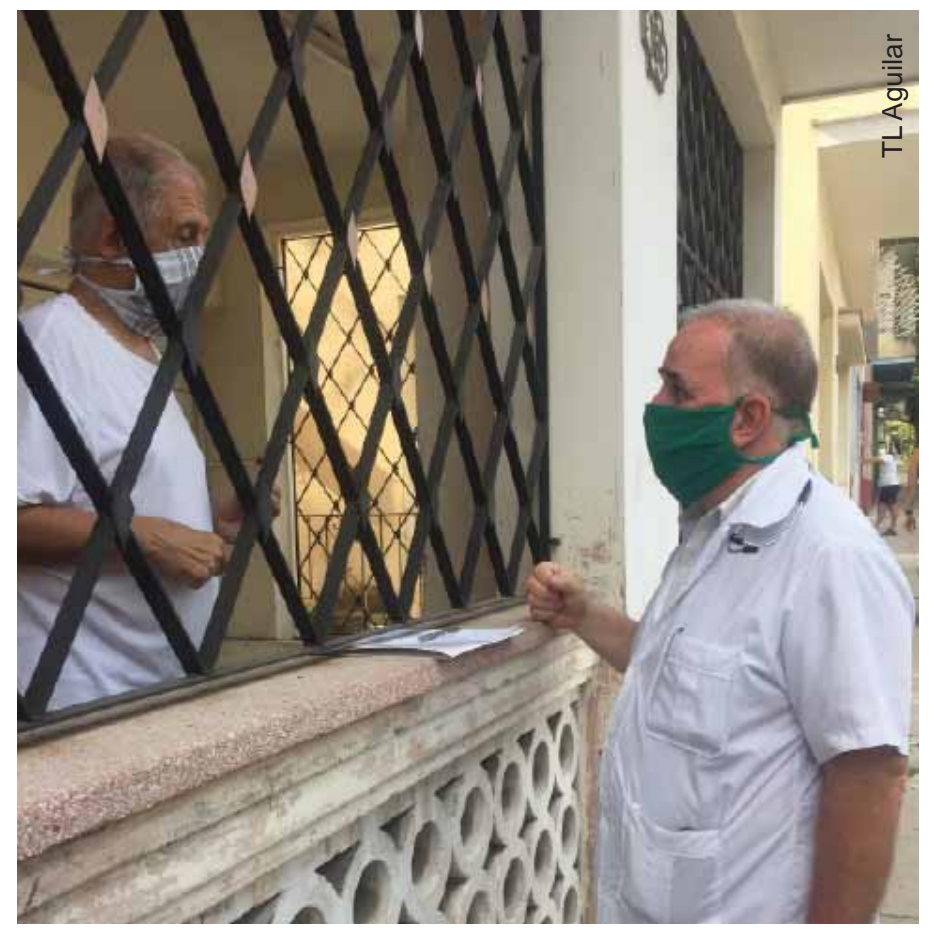

Dr Fadragas: "You feel a tremendous sense of responsibility."

Family doctors and nurses are literally next door, their communities listen to them, and they count on their communities: This two-way street may be the most critical, long-lasting effect of this Cuban strategy relying so much on primary care: since these health professionals are embedded in their communities, they have gained a solid reputation over time, are respected and listened to. This makes them better at influencing local residents than just about anybody else, and they are also there to see when people aren't abiding by physical distancing, sheltering at home, or when an older resident living alone needs a helping hand from a neighbor.

And certainly not all is well concerning risk perception and individual responsibility, especially in densely populated, high-risk municipalities like Plaza. Comments Dr Rubén García: "I think at one point, people started becoming too confident, when they saw the number of cases wasn't rising very fast. When there were fewer travelers coming from abroad, too. Right now, I think the main problem we have is respect for physical distancing, people on the street without face masks. People who don't have to be 
there, unnecessary congestion, even though we realize people have to shop for basic needs. As a member of the local Civil Defense Council, I've brought that up, and we're working on it. This is where contributions from other sectors become so important."

Dr Fadragas notes: "When we see people in the street not correctly using their face masks, or not using them at all, then family doctors and nurses have the capacity to influence them, to convince them how important it is to follow these guidelines, to be more responsible. And when we find an elderly person outside, we not only speak with them, but also with their family, to make them understand how risky that is."

Not all family doctors stay as long in one neighborhood as Drs Fadragas and Gálvez, and turnover in some areas of Havana is high. Dr Fadragas says "when you have spent 18 years in a place like I have, people respect you and trust you, not just because you're always there, but because they assess your work with them and their families over time. So we, along with the nurses in our teams, have an opportunity like no others to modify people's behavior for their own health...critical at this juncture with COVID-19."

Local organizations, such as the Federation of Cuban Women and seniors' clubs are active in helping family doctors and nurses, especially when it comes to additional attention to older adults living alone, until the system kicks in that involves social workers taking them meals and other necessities. Small business owners have also come together in a network called Covidvoluntarios to shop for elderly who live alone, as well as for disabled residents in their neighborhoods.

Nurses are the linchpins in many neighborhoods, especially where doctors may frequently change. "A nurse's role is fundamental," says Josefa Nietos. "We are the communicators, the educators, and we're there so that people feel accompanied and more secure. In these times of COVID-19, people are afraid they may be exposed to this virus, and we're there to calm their fears, and constantly remind them what they can do to protect themselves and their families."

"We talk a lot," she says, "because we need to talk a lot. Even before the first cases were diagnosed, we held neighborhood meetings here to explain to people what this pandemic means, what the symptoms are, and what they needed to do. Then we started going door-to-door with the medical students, repeating the same messages. Going to people's homes also meant fewer patients coming into our office, thus better physical distancing. Luckily, Cubans are educated, and they can understand the reasons why we're calling on them to be more responsible."

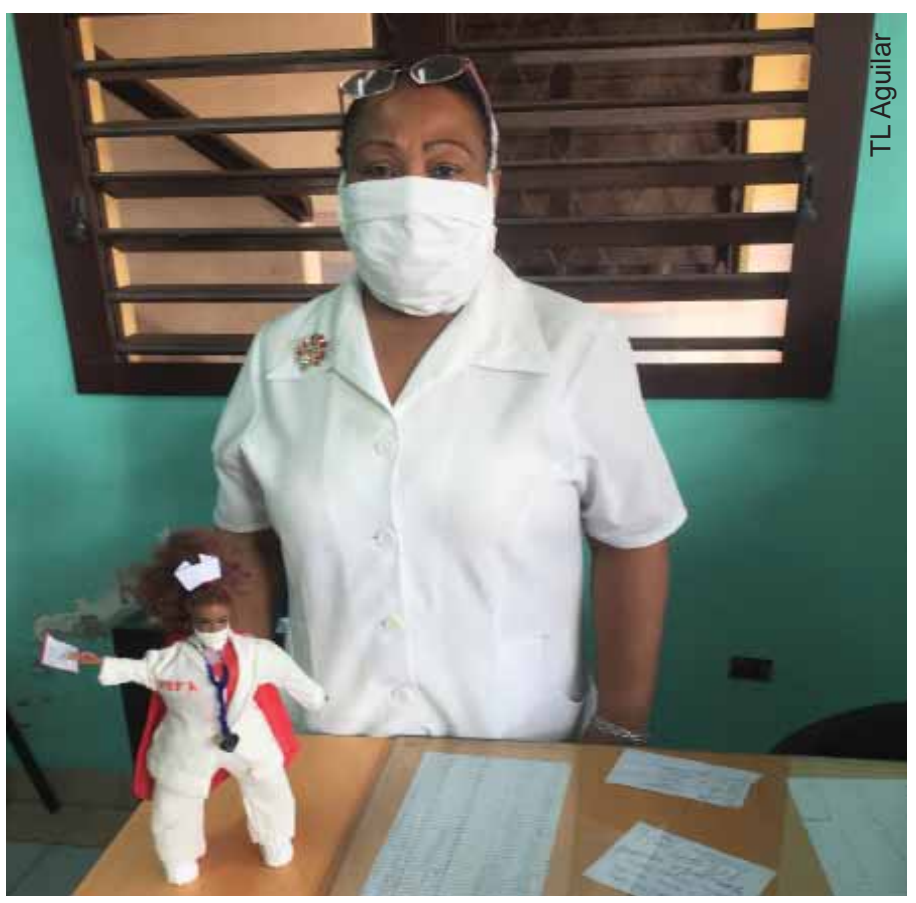

Nurse Josefa Nietos: "The children were given a homework assignment to represent who was their 'superhero' in the fight against COVID-19, and this young girl decided it was me, her nurse!"

Community cooperation is also one way of expressing community appreciation for these primary care professionals. Another, of course, is the evening rounds of applause heard now in almost every country. But in Cuba, there is no doubt that the doctors and nurses are right there in the community to hear it for themselves. -1 -

\section{Notes \& References}

1. Lewis C, Seervai, Shah T, Abrams MK, Zephyrin L. Primary Care and the COVID-19 Pandemic [Internet]. New York: The Commonwealth Fund blog 2020 Apr 22 [cited 2020 May 1]. Available at: https://www.commonwealthfund. org/blog/2020/primary-care-and-covid-19-pandemic

2. Daily Cuban Ministry of Public Health. Press conference on COVID/19 situation in Cuba offered by Dr Francisco Durán, National Director of Epidemiology, 2020 May 1.

3. NY Times [Internet]. New York: New York Times; c2020. The Coronavirus Outbreak. Maps and Tracker. Coronavirus Map: Tracking the Global Outbreak; 2020 May [updated 2020 May 3; cited 2020 May 1]. Available at: https://www .nytimes.com/interactive/2020/world/coronavirus-maps.html

4. National Health Statistics and Medical Records Division (CU). Anuario Estadístico de Salud 2018. Havana: Ministry of Public Health (CU); 2019. p. 122. Spanish.

5. See Cuba's COVID-19 Strategy: Main Actions Through April 23, 2020, this issue.

6. See COVID-19 Case Detection: Cuba's Active Screening Approach, this issue. 